\title{
Análise sobre a gamificação em Ambientes Educacionais
}

\author{
Aline Nunes Ogawa, Gabriel Galdino Magalhães, \\ Ana Carolina Tomé Klock, Isabela Gasparini \\ Departamento de Ciência da Computação - PPGCA e PPGECMT \\ Universidade do Estado de Santa Catarina (UDESC) - Joinville, SC - Brasil \\ alinenogawa@hotmail.com, \{actklock, gbljacu\}@gmail.com, \\ isabela.gasparini@udesc.br
}

Resumo. A gamificação é a utilização de elementos de jogos em outros contextos sem relação com jogos, sendo uma técnica bastante utilizada nos ambientes educacionais para motivar os alunos. O objetivo desse trabalho é identificar como é feita a avaliação da experiência e interação do usuário com a gamificação aplicada em ambientes educacionais. Foram analisados trabalhos que fazem experimentos controlados comparando um ambiente educacional com e sem gamificação. Nesses trabalhos, existe uma grande variação em relação ao contexto, ao tempo de duração, ao número de participantes e de elementos utilizados. As métricas empregadas foram obtidas através de implementações no ambiente e em questionários de satisfação. Espera-se que, com o levantamento de diferentes formas de experimentação, outros trabalhos possam avaliar a utilização da gamificação em ambientes educacionais.

Palavras-chave: gamificação, ambientes educacionais, avaliação.

Abstract. Gamification is the use of game elements in other contexts unrelated to games, and is a technique widely used in the educational environments to motivate students. The aim of this work is identify how user experience and user interaction with gamification in educational environments is evaluated. We analyzed works that performed controlled experiments comparing an educational environment with and without gamification. In these works, there is a wide variation in relation to the context, the duration, the number of participants and elements used. The metrics applied were obtained through implementations in the environment and satisfaction questionnaires. With the different forms of experiments, other works can evaluate the use of gamification in educational environments.

Keywords: gamification, educational environments, evaluation.

\section{Introdução}

Atualmente existe um grande investimento na área de informática na educação, visando encontrar diferentes tecnologias que possam ser aplicadas para auxiliar nos processos de ensino e de aprendizagem. A educação é uma área que necessita de novas estratégias para atrair os alunos, mas os mesmos se mostram desinteressados pelos métodos passivos de ensino e aprendizagem utilizados na maioria das escolas (Fardo, 2013). Uma tecnologia que vem sendo amplamente estudada e aplicada na área educacional é a gamificação.

A gamificação pode ser resumida como o uso de elementos de jogos em contextos não relacionados com jogos (Deterding et al., 2011). Para auxiliar o estudante a alcançar seu objetivo (aprender), a gamificação pode aumentar progressivamente a dificuldade dos exercícios, permitir que o estudante cometa erros (o que não é bem tolerado na educação tradicional) e promover competições e colaboração entre os estudantes a fim de ensinar ao mesmo tempo em que diverte (Fardo, 2013). Entretanto, para que a gamificação produza o efeito esperado, é necessário considerar o conteúdo a 
ser aprendido, as características dos estudantes e as tarefas disponíveis no ambiente virtual de aprendizagem (AVA). Desta forma, diferentes trabalhos têm avaliado o efeito da gamificação em ambientes educacionais, comparando o mesmo ambiente gamificado com o tradicional.

Este artigo tem como objetivo identificar como é realizada a comparação de ambientes com gamificação e sem gamificação. Para alcançá-lo, foram analisados diferentes trabalhos para verificar como é realizado o processo de avaliação dos ambientes, quais as métricas utilizadas, qual o tempo de duração, o número de participantes e de elementos utilizados. Este trabalho está estruturado da seguinte forma: o conceito e os fundamentos sobre gamificação são descritos na seção 2. Em seguida, são explorados os trabalhos que comparam a utilização de sistemas com gamificação e sem gamificação na seção 3, enquanto a seção 4 faz a análise comparativa entre esses trabalhos. Por fim, a seção 5 apresenta as conclusões do artigo.

\section{Fundamentos sobre Gamificação}

A gamificação (do inglês Gamification) é o termo utilizado para definir a utilização de elementos e design de jogos em contextos não lúdicos a fim de estimular e motivar as pessoas a alcançarem algum objetivo específico, como uma mudança de comportamento ou o desenvolvimento de novas habilidades (Werbach; Hunter, 2012; Deterding et al., 2011).

\subsection{Técnicas de gamificação}

$\mathrm{Na}$ área educacional, a gamificação contém um amplo conjunto de abordagens de ensino e de aprendizagem e, quando aplicada de forma eficaz, pode ajudar com a aquisição de uma nova habilidade ao mesmo tempo em que aumenta a motivação para aprender (Johnson et al., 2013).

Entre os elementos de jogos comumente utilizados em AVAs, podem-se citar: feedback constante, desafios, competição, conquistas, recompensas, pontos, medalhas, missões, personalização, regras, narrativa, níveis e rankings (Bissolotti; Nogueira; Pereira, 2014; Klock et al., 2014). A maioria dos elementos de jogos aplicados na gamificação está diretamente relacionada aos desejos humanos, por exemplo: pontos são conectados com a necessidade de recompensa; níveis são úteis para demonstrar status; desafios permitem alcançar realizações; rankings estimulam a competição; presentes permitem que as pessoas pratiquem a solidariedade (altruísmo); entre outros (BBVA Innovation Edge, 2012).

Em um ambiente educacional, uma mesma disciplina pode ter diferentes estudantes que, por sua vez, possuem diferentes características (e.g., pessoais, pedagógicas, culturais), podendo influenciar na melhora do aprendizado do estudante. Portanto, é necessário entender quem são os usuários dos AVAs e o contexto em que eles estão inseridos para que a gamificação possa realmente trazer os benefícios esperados para a área educacional.

\section{Trabalhos levantados}

Através uma busca exploratória em mecanismos de busca acadêmicos, foi encontrada na literatura trabalhos que avaliam e comparam a experiência do estudante em AVAs utilizando experimentos controlados antes da implementação das técnicas de gamificação e após a implementação das mesmas. Entre esses trabalhos, destacam-se: Halan et al. (2010), Li et al. (2012), Denny (2013), De-Marcos et al. (2014) e Vassileva et al. (2015), descritos nas próximas subseções. 


\subsection{Halan et al. (2010)}

O trabalho de Halan et al. (2010) propõe e avalia a aplicabilidade de algumas técnicas de gamificação (e.g., rankings, narrativas e desafios) como estratégia de motivação para persuadir os estudantes de medicina à modelarem a conversação de seres humanos virtuais (nesse caso, modelando pacientes virtuais). $\mathrm{O}$ estudo teve um design experimental between-subjects (entre-sujeitos) com o uso da motivação externa como fator independente. Os vinte participantes foram divididos aleatoriamente em dois grupos: o primeiro grupo, composto por nove participantes, serviu para controle e teve acesso ao sistema sem os elementos da gamificação, enquanto o segundo grupo, composto por onze participantes, serviu para a experimentação e teve acesso ao sistema gamificado.

Os autores trabalharam com duas hipóteses: H1) Os participantes que utilizaram o sistema gamificado estão mais propensos a ter interações com o sistema do que os participantes que utilizaram o sistema sem gamificação e; H2) Os participantes que utilizaram o sistema gamificado passam uma quantidade maior de tempo interagindo com o sistema do que os participantes que utilizaram o sistema sem gamificação. Para testá-las, Halan et al. (2010) utilizaram as seguintes métricas: o número de interações (considerando o número total de interações do participante com o sistema) e a duração dessas interações (considerando o tempo desde o início da interação até quando o participante conclui a ação).

Através de uma análise ANOVA one-way do número de interações, foi demonstrado que a participação dos estudantes teve uma diferença significativa ( $\mathrm{p}<$ 0,001 ) entre os dois grupos. Já com uma análise Tukey HSD, foi possível observar um número significativamente maior de interações dos participantes do grupo experimental em relação aos participantes do grupo de controle $(\mathrm{p}<0,01)$. Assim, Halan et al. (2010) aceitaram a hipótese H1. Outro resultado apresentado foi em relação à duração das interações, onde a diferença em tempo médio das interações entre o grupo experimental e o grupo de controle não atingiram significância estatística $(\mathrm{p}=0,435)$. Isso pode indicar que os participantes não passaram mais tempo no sistema gamificado e, portanto, os resultados não suportam a hipótese H2. Outros experimentos devem ser aplicados para verificar essa hipótese.

Além desses resultados, Halan et al. (2010) apresentaram as diferenças de abordagem do usuário com a interação, onde os participantes do grupo experimental tiveram um comportamento menos realista durante a interação, avaliado através do uso de frases de saudação nas interações. Enquanto $82 \%$ dos participantes no grupo de controle utilizaram frases como "Oi" ou "Como está" na interação, somente $11 \%$ dos participantes do grupo experimental utilizavam frases de saudação nas interações. Os autores usaram um teste de Fischer two-tailed com resultado significativo $(\mathrm{p}<0,001)$. Tal resultado pode indicar que a motivação do participante estava em apenas obter uma pontuação alta ao invés de realizar uma entrevista médica realista.

Apesar do trabalho de Halan et al. (2010) não classificar sua metodologia e coleta de dados (quanto a ser qualitativa, quantitativa ou ambas), foi possível notar que os autores utilizaram ambas abordagens, quantitativamente calculando o número de interações e a duração das interações dos usuários no sistema (que podem ser considerados como resultados comportamentais) e qualitativamente, considerando a abordagem dos usuários no sistema (considerados como resultados psicológico e comportamental da gamificação). As implementações que registraram as interações dos 
participantes e o tempo das interações foram os instrumentos utilizados para realizar a avaliação.

\subsection{Li et al. (2012)}

O trabalho de Li et al. (2012) apresenta o GamiCAD, um sistema tutorial interativo gamificado para novos usuários do AutoCAD. O GamiCAD oferece feedbacks de áudio e vídeo em tempo real, além de reconhecer os erros e acertos do usuário de acordo com sua progressão nas atividades. Li et al. (2012) fizeram uma avaliação empírica do GamiCAD comparando-o com um sistema tutorial equivalente, porém sem os elementos da gamificação.

O objetivo do estudo comparativo apresentado foi responder a duas questões principais: 1) Se a inclusão dos elementos de gamificação fornece uma experiência de aprendizagem mais envolvente e 2) Se um sistema tutorial gamificado tem um impacto positivo na aprendizagem. Para tal, foi levada em consideração a hipótese de que os elementos da gamificação aumentam os níveis de engajamento do usuário e, por sua vez, impactam positivamente na aprendizagem.

O experimento analisou dados obtidos de catorze participantes (dez mulheres e quatro homens), recrutados através de anúncios on-line, com idades entre dezenove e sessenta e dois anos e sem nenhuma experiência no AutoCAD, divididos em dois grupos: um de controle e um experimental. As medidas quantitativas coletadas para o estudo foram: a taxa de conclusão das tarefas propostas e o tempo para os participantes concluírem cada uma delas.

Foi realizada uma análise das taxas de conclusão das tarefas: a taxa foi de $100 \%$ para o sistema gamificado, enquanto a taxa para o sistema sem gamificação foi de $90,5 \%$. Os autores, entretanto, não mostraram como esse resultado foi validado. Ao calcular os tempos de conclusão das tarefas, Li et al. (2012) atribuíram uma duração máxima de 480s para qualquer tarefa que o participante não tenha conseguido concluir. Com isso, os tempos médios de conclusão das tarefas foram 111s para o sistema gamificado e 261s para o sistema sem gamificação. Um teste t de amostras pareadas (paired sample t-test), que é um teste paramétrico usado para comparar duas amostras independentes, mostrou que essa diferença foi significativa para a maioria das tarefas.

Após a conclusão do estudo, foi aplicado um questionário de satisfação para coletar dados qualitativos dos participantes. Os participantes foram convidados a avaliar o sistema utilizado por meio de oito perguntas em uma escala de Likert de 5 pontos (sendo 1 como "Discordo Plenamente" e indo até 5, "Concordo Plenamente"). Os autores utilizaram o teste $\mathrm{U}$ de Mann-Whitney para avaliar se a diferença das respostas foi realmente significativa, mas apenas cinco das questões tiveram diferenças significativas nas repostas $(\mathrm{p}<0,05)$. Em geral, os participantes consideraram que $\mathrm{o}$ sistema gamificado era mais agradável, divertido, envolvente e eficaz do que o sistema sem a gamificação. Além disso, o sistema tutorial sem gamificação recebeu alguns feedbacks negativos. Na avaliação, os participantes que utilizaram o sistema gamificado relataram níveis maiores de engajamento, foram os mesmos que executaram de forma mais rápida o conjunto de tarefas proposto e tiveram taxa de conclusão superior aos participantes que utilizaram o sistema sem gamificação.

\subsection{Denny (2013)}

O trabalho de Denny (2013) relata um experimento controlado realizado em 2012 para medir o impacto dos elementos de gamificação em uma ferramenta de aprendizagem online, o PeerWise. Esse experimento foi aplicado ao curso POPLHLTH111 da 
Universidade de Auckland (Nova Zelândia), que apresentou aos estudantes estruturas e ferramentas para compreender e controlar o impacto de doenças nas populações. Esse curso já vinha sendo utilizado pela universidade no PeerWise (sem gamificação) desde 2008.

Tal curso foi escolhido por apresentar uma grande quantidade de alunos, que foram divididos em dois grupos aproximadamente iguais: um de controle (com 515 alunos) e um experimental (com 516 alunos). Todos os aspectos do sistema foram trabalhados de forma idêntica para ambos os grupos, exceto pelo fato de o grupo experimental ter acesso ao sistema de medalhas. $\mathrm{O}$ experimento foi realizado em quatro semanas do curso.

O objetivo desse experimento foi descobrir se os elementos de gamificação aplicados no PeerWise têm um impacto sobre o número de perguntas e respostas realizadas pelos estudantes e o número de dias distintos que um estudante se envolveu com a ferramenta. Portanto, foram definidas três medidas de participação: número de perguntas de autoria do estudante, o número de respostas apresentadas pelo mesmo (que refletem na funcionalidade principal do PeerWise) e o número de dias distintos que o estudante utilizou a ferramenta. Foram considerados como dia de acesso os dias em que o estudante fez ou respondeu a alguma pergunta.

Durante o experimento, quinze alunos do grupo de controle e doze alunos do grupo experimental não participaram do PeerWise. Portanto, foram considerados grupos de 500 e 504 alunos (respectivamente) que criaram ou responderam alguma pergunta no ambiente, indicando uma alta taxa de participação dos alunos $(97 \%)$ em relação ao grupo inicial. Para determinar a significância das diferenças entre os grupos, foram realizados testes de Mann-Whitney. A distribuição do número de perguntas feitas não diferiu significativamente entre os dois grupos, sendo quase idênticas ( $U=131374,5, \mathrm{z}$ $=0,31, \mathrm{p}=0,7566)$, mas, a distribuição do número de respostas apresentadas por aluno diferiu significativamente entre os dois grupos (Mann-Whitney $U=116.386,5, \mathrm{Z}=$ $3,45, \mathrm{p}<0,001$ ), sendo que o número médio de respostas dos alunos do grupo de controle foi de 60 respostas, enquanto o grupo experimental foi de 68 respostas. A distribuição do número de dias ativos no sistema também diferiu significativamente entre os dois grupos (Mann-Whitney $U=116584,5, Z=3,41, p<0.001$ ), sendo que o número médio de dias ativos para o grupo de controle foi de 6,21 , enquanto no grupo experimental a média chegou a 7,01.

Além dos dados quantitativos, foi realizada uma pesquisa qualitativa com todos os alunos para responder perguntas demográficas, de conhecimento do conteúdo e sobre o ambiente PeerWise. Para os alunos do grupo experimental, também foi solicitado que informassem sua experiência prévia com o elemento de jogo disponibilizado no ambiente (i.e., medalhas) e se a utilização do mesmo influenciou na sua motivação. Tal como Li et al. (2012), esses dados foram obtidos com uma escala de Likert de 5 pontos. Nessa pesquisa, estudantes de ambos os grupos foram positivos em relação à aprendizagem ( $83 \%$ dos estudantes indicaram que é benéfico para o estudante ser autor de suas próprias perguntas e $80 \%$ também consideraram importante responder as questões dos colegas de classe). Em relação às medalhas, houve também uma melhoria da experiência do estudante no ambiente ao utilizá-las (onde $60 \%$ dos estudantes afirmaram que a possibilidades de ganhar medalhas era agradável e que preferiam utilizar o PeerWise com medalhas).

Uma preocupação dos instrutores do curso é que a gamificação pudesse aumentar o número de perguntas e respostas do grupo experimental, mas com redução 
da qualidade das mesmas, tal como no experimento de Halan et al. (2010), onde o resultado das abordagens indicou que a motivação do participante estava em realizar uma quantidade alta de interações e receber uma pontuação por isso, ao invés de estar em realizar uma entrevista médica realista. Mas isso não ocorreu durante o experimento de Denny (2013), pois não houve diferença significativa entre os dois grupos em termos de exatidão das respostas apresentadas. Além disso, foi possível observar um aumento do período de tempo do acesso, apesar de não apresentar diferença significativa na quantidade de perguntas criadas.

Através do exposto, foi possível verificar que o autor utilizou metodologias quantitativas e qualitativas para a coleta e análise dos dados no experimento e que as suas métricas se relacionavam com resultados comportamentais como também psicológicos.

\subsection{De-Marcos et al. (2014)}

O trabalho de De-Marcos et al. (2014) descreve uma avaliação empírica em uma turma de Tecnologias de Informação e Comunicação utilizando três versões de um ambiente educacional: uma tradicional, uma gamificada e uma rede social. $\mathrm{O}$ curso tem duração de quinze semanas e utiliza uma abordagem onde os estudantes têm três horas de aula por semana e tais aulas são complementadas com leituras e atividades a serem entregues através de um sistema de gestão de aprendizagem chamado Blackboard. A avaliação do desempenho do estudante no curso é feita com a aplicação de quatro atividades práticas e uma prova final teórica.

Para o experimento, os estudantes foram separados aleatoriamente em três grupos: o grupo de controle (que utilizou o sistema tradicional) possuía 73 estudantes, o grupo experimental de gamificação possuía 114 estudantes e o grupo experimental de rede social possuía 184 estudantes. Durante o experimento, os materiais e as atividades foram entregues a todos os grupos de forma idêntica através do ambiente educacional e durante as aulas. Os experimentos foram executados e os dados quantitativos sobre o desempenho e participação dos estudantes foram recolhidos durante o semestre letivo. Entretanto, os estudantes dos grupos experimentais podiam também utilizar o sistema tradicional.

O objetivo dessa avaliação era comparar as duas outras abordagens com a tradicional no mesmo local de estudo para determinar a sua eficácia em termos de desempenho dos estudantes, níveis de participação, engajamento e atitudes dos estudantes em relação a cada ferramenta. Assim, três questões foram trabalhadas: se a gamificação e/ou as redes sociais impactam na aprendizagem dos estudantes em ambientes educacionais, se as mesmas impactam nas taxas de participação e também se os estudantes têm uma atitude positiva perante essas ferramentas.

Pré-testes e pós-testes foram usados para avaliar o desempenho dos estudantes. A pontuação de participação foi automaticamente calculada com base na participação nas aulas e contribuições para o curso. Todos os resultados foram normalizados para uma escala de $0-100$. Resultados do pré-teste analisados utilizando o teste de variância ANOVA one-way sugeriram que não havia diferença significativa entre os grupos de controle e experimental inicialmente. Resultados do pós-teste sugeriram que ambos os grupos experimentais superaram o grupo de controle nos quatro trabalhos práticos. No entanto, os estudantes do grupo de controle superaram ambos os grupos experimentais no exame escrito final, além de terem superado os estudantes do grupo da gamificação e 
os estudantes do grupo de rede social em pontos de participação. Essas diferenças foram estatisticamente significativas.

Já a questão atitudinal foi obtida através de um questionário com dez perguntas, também com uma escala de Likert de 5 pontos, para recolher dados quantitativos e qualitativos e identificar o nível de satisfação e a percepção dos estudantes sobre os instrumentos experimentais (gamificação/rede social). As respostas dos estudantes indicaram que as atitudes em relação às duas experiências (i.e., gamificação e rede social) foram positivas. Dos 45 estudantes do grupo experimental de gamificação que responderam o questionário, teve-se uma média de satisfação de 3,64 pontos e, dos 97 estudantes do grupo experimental de rede social, teve-se uma média de satisfação de 3,78 pontos.

Como resultado, pode-se observar que ambas as abordagens apresentaram melhora no desempenho dos estudantes em relação a uma abordagem tradicional para ambientes educacionais em termos de desempenho acadêmico para trabalhos práticos. Em contraste, a utilização do ambiente tradicional se apresentou superior em termos de participação e desempenho na prova teórica. Os autores utilizaram metodologias quantitativas para a coleta e análise das métricas de desempenho e participação e qualitativas para as métricas de percepção e satisfação dos estudantes. As métricas analisadas se relacionavam com resultados comportamentais e psicológicos.

\subsection{Vassileva et al. (2015)}

O trabalho de Vassileva et al. (2015) propõe um estudo para investigar se é possível reduzir comportamentos indesejáveis e aumentar o desempenho dos estudantes através do uso da gamificação. Para isso, foi desenvolvido um ambiente educacional gamificado, o E-Game, que premia o desempenho bem-sucedido dos estudantes com vários elementos de jogos (e.g., pontos, medalhas e níveis). $\mathrm{O}$ E-Game foi projetado para ser um ambiente de apoio ao ensino de matemática e ao processo de aprendizagem à distância. Além disso, o E-Game suporta upload de vídeos para que o professor possa adicionar ajudas aos estudantes durante as suas tarefas.

A partir disso, um estudo de caso foi realizado para investigar o comportamento durante a interação com o E-GAME com dois grupos de estudantes: um grupo de controle (sem gamificação) e um grupo experimental (com gamificação). O objetivo do estudo era verificar se elementos de gamificação aumentam a motivação e ajudam a desencorajar os comportamentos indesejados do estudante durante a utilização do ambiente educacional e se a mecânica do jogo aumenta o desempenho de aprendizagem dos estudantes durante a utilização desse ambiente.

O estudo de caso foi realizado em uma escola do ensino fundamental e médio em Saskatoon (Canadá), com uma classe de dezesseis estudantes da sétima série (com idades entre doze e treze anos) que estavam usando o ambiente educacional E-Game. A turma foi dividida em dois grupos de oito estudantes de forma aleatória, sendo que um dos grupos utilizou o ambiente educacional gamificado (experimental) e outro grupo utilizou o ambiente sem gamificação. Conscientes de que a amostra é muito pequena para conseguir obter significância estatística e generalizada, os autores apresentaram que o estudo de caso tem principalmente um propósito exploratório, buscando testar hipóteses preliminares e descobrir as influências que gamificação possa ter sobre o desempenho e motivação do aluno.

O trabalho de Vassileva et al. (2015) tem o objetivo responder se há uma diferença significativa em termos de motivação, se há um redução de comportamentos 
indesejáveis em um ambiente educacional gamificado entre meninos e meninas e se há uma diferença significativa em termos de desempenho em um ambiente educacional gamificado entre meninos e meninas. As métricas utilizadas para descobrir tais respostas foram: a pontuação obtida pelos alunos durante a utilização do E-Game e a motivação dos alunos, obtida através de um questionário aplicado ao final do experimento. Assim, foi realizado um teste $\mathrm{t}$ de amostras pareadas (paired sample $t$ test), como no experimento de $\mathrm{Li}$ et al. (2012). Com os testes, não ficou claro estatisticamente se o ambiente gamificado é mais útil no sentido de motivação do que o ambiente não gamificado (não respondendo a primeira pergunta). Apesar da maioria dos resultados não serem estatisticamente significativos (devido ao tamanho da amostra), foi importante executar uma análise estatística para identificar tendências nos dados e para fornecer informações que auxiliem no uso correto dos elementos de jogos em ambientes de aprendizagem. Entretanto, para a segunda pergunta, os autores encontraram evidências estatisticamente significativas de que os meninos tiveram um melhor desempenho no ambiente com gamificação do que as meninas.

Os resultados indicaram que a gamificação implementada no E-Game contribuiu para melhorar o desempenho dos estudantes do sexo masculino, mas não das estudantes do sexo feminino. Além disso, não foi possível concluir se o uso da gamificação ajuda a prevenir o comportamento inadequado dos estudantes. Os autores utilizaram metodologias quantitativas para coletar e analisar os dados de desempenho e metodologias qualitativas para a coleta e análise da satisfação dos estudantes, sendo essas métricas relacionadas com resultados comportamentais e psicológicos respectivamente.

\section{Análise Comparativa}

Através do exposto, foi possível notar que os experimentos realizados utilizaram a gamificação em contextos diversificados, abordando desde o aprendizado no ensino fundamental, como no trabalho de Vassileva et al. (2015) que utilizou um ambiente para ensino de matemática com estudantes da sétima série, até um aprendizado especializado, como o trabalho de Halan et al. (2010) que utilizou um ambiente para melhorar o aprendizado de universitários da área da saúde.

A quantidade de estudantes utilizada nos experimentos também se mostrou bastante diversificada, sendo que apenas um dos trabalhos utilizou mais de 1.000 estudantes (Denny, 2013), seguido pelo trabalho de De-Marcos et al. (2014) que teve a participação de 371 estudantes, Halan et al. (2010) que realizou seu experimento com 20 estudantes, Vassileva et al. (2015) com 16 estudantes e Li et al. (2012) com apenas 14 estudantes. Outro fator que se apresentou variado, apesar de somente dois dos autores fornecerem essa informação, foi o tempo de duração das coletas de dados dos experimentos, onde Denny (2013) avaliou somente quatro semanas de uso do ambiente e De-Marcos et al. (2014) avaliou quinze semanas de uso do ambiente no curso.

Dos trabalhos apresentados, apenas um dos experimentos avaliou um único elemento de gamificação, que foi o caso do trabalho de Denny (2013) que apresentou um ambiente educacional gamificado apenas com medalhas, enquanto os outros trabalhos inseriram uma quantidade maior de elementos de gamificação no ambiente avaliado, como o trabalho de Li et al. (2012) que apresentou um ambiente com: narrativa, objetivos, feedback, recompensas, progressão e desafios.

As métricas utilizadas variaram entre resultados comportamentais e psicológicos. As métricas comportamentais foram medidas com implementações 
realizadas pelos pesquisadores e focaram em analisar principalmente: a quantidade, o tempo de duração e a taxa de conclusão das interações no sistema, as notas, o número de perguntas feitas e respondidas pelos estudantes no ambiente. Já para as métricas psicológicas, houve um enfoque maior em aspectos como a motivação, a atitude, a percepção e a satisfação dos alunos ao utilizar o ambiente. Essas métricas foram coletadas e analisadas utilizando questionários e pesquisas de satisfação com os alunos.

Dos cinco trabalhos citados, apenas um (Halan et al., 2010) não definiu as questões de pesquisas que foram trabalhadas durante o experimento. Embora não explicitamente afirmadas, a questão de pesquisa genericamente compartilhada entre os trabalhos foi: "Será que a gamificação funciona?". Apenas dois dos trabalhos não apresentaram hipóteses para a avaliação, que foi o caso de Denny (2013) e De-Marcos et al. (2014). As técnicas de significância estatística utilizadas pelos trabalhos também se distinguiram. No entanto, as principais validações foram feitas utilizando: ANOVA one-way, Tuckey HSD, Paired Sample T-Teste e Teste U de Mann-Whitney. Dentre os trabalhos analisados, foi possível perceber que todos seguem uma mesma ordem cronológica, composta pelas fases apresentadas na Figura 1.

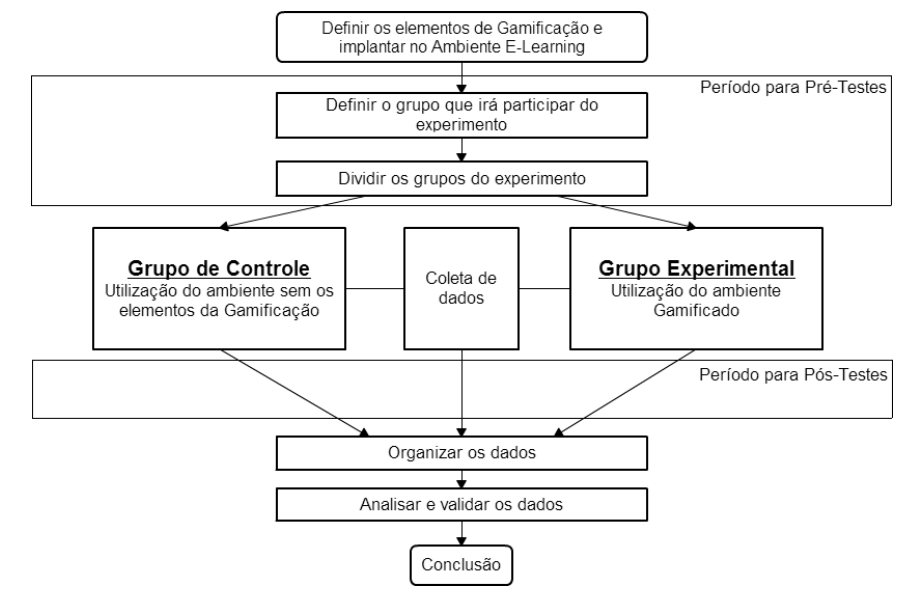

Figura 1. Fluxograma das etapas presente nos experimentos levantados

\section{Conclusão}

A gamificação é uma ferramenta que tem sido utilizada para auxiliar na motivação e engajamento das pessoas a alcançarem um objetivo específico. No ambiente educacional, a gamificação permite que o estudante se divirta enquanto aprende, tornando o processo de ensino-aprendizagem mais engajador, motivador e atraente para estudantes e professores. Existem diversos trabalhos que avaliam o processo de gamificação, sendo que cada um aplica seu processo de avaliação, utilizando diferente métricas e elementos de gamificação. A análise comparativa dos trabalhos identificou os diferentes contextos em que foram realizados os experimentos, a variação do número de participantes e de elementos avaliados, as métricas utilizadas e a maneira como elas foram obtidas, analisadas e validadas. Foi possível identificar e elaborar um fluxograma das etapas presente nos experimentos, que são: definir os elementos de gamificação e implantar no sistema, definir o grupo de interesse para aplicar o experimento, dividir esse grupo de pesquisa em um grupo de controle e grupo(s) experimental(ais), realizar pré-testes (que podem ser realizados tanto antes como depois da divisão dos grupos), a utilização do sistema pelos grupos em separado e coleta dos dados de uso, que posteriormente podem ser acrescentados de pós-testes e, por fim, a organização e análise dos dados do obtidos no experimento. 
Os educadores reconhecem que o design de jogos pode estimular ganhos de produtividade e criatividade entre os estudantes (Johnson et al., 2014), premiando-os pela dedicação e eficiência. Além disso, proporciona ao estudante um sistema que permite visualizar o efeito de suas ações e aprendizagem, facilitando a compreensão da relação das partes com o todo. Porém, é necessário conhecer os alunos, o conteúdo a ser apresentado e o ambiente em que será inserido para que seja obtido o resultado esperado. Entre os trabalhos futuros, pretende-se avaliar o ambiente educacional AdaptWeb $^{\circledR}$ gamificado versus o não gamificado, verificando a motivação $\mathrm{e}$ engajamento do estudante com o ambiente.

\section{Referências}

BBVA INNOVATION EDGE. Gamification: The business of fun. Madrid: BBVA Innovation Center, 2012.

BISSOLOTTI, K.; NOGUEIRA, H. G.; PEREIRA, A. T. C. "Potencialidades das mídias sociais e da gamificação na educação a distância". In: RENOTE - Revista de Novas Tecnologias na Educação, v. 12, no. 2, 2014.

DE-MARCOS, L.; DOMÍNGUEZ, A.; SAENZ-DE-NAVARRETE, J.; PAGÉS, C. "An empirical study comparing gamification and social networking on e-learning". Computers \& Education, 75, 2013, 82-91.

DENNY, P. "The Effect of Virtual Achievements on Student Engagement". In Proceedings of CHI 2013: Changing Perspectives, April 27-May 2, 2013, Paris, France, pp. 763-772.

DETERDING, S.; DIXON, D.; KHALED, R.; NACKE, L. "Gamification: Toward a definition". In: Proceedings of the 2011 Conference on Human Factors in Computing Systems, Association for Computing Machinery, 2011.

FARDO, M. F. “A gamificação aplicada em ambientes de aprendizagem”. In: RENOTE Revista de Novas Tecnologias na Educação, v. 11, no. 1, 2013.

HALAN, S.; ROSSEN, B.; CENDAN, J.; LOK, B. "High Score! Motivation Strategies for User Participation in Virtual Human Development". In Proceedings of the international conference on Intelligent Virtual Agents, 2010, Springer Berlin/Heidelberg, pp. 482-488.

JOHNSON, L.; ADAMS, S.; CUMMINS, M.; ESTRADA, V.; FREEMAN, A.; LUDGATE, H. The NMC Horizon Report: 2013 Higher Education Edition. New Media Consortium, 2013.

JOHNSON, L.; BECKER, S. A.; BROWN, M. The NMC Horizon Report: 2014 Higher Education Edition. New Media Consortium, 2014.

KLOCK, A. C. T.; CARVAlHO, M. F.; ROSA, B. E.; GASPARINI, I. "Análise das técnicas de Gamificação em Ambientes Virtuais de Aprendizagem". In: RENOTE Revista de Novas Tecnologias na Educação, v. 12, no. 2, 2014.

LI, W.; GROSSMAN, T.; FITZMAURICE, G. "GamiCAD: a gamified tutorial system for first time autocad users". In Proceedings of the 25th annual ACM symposium on User interface software and technology, October 7-10, 2012, Cambridge, Massachusetts, USA, ACM, pp. 103-112.

SMITH-ROBBINS, S. This Game Sucks: How to Improve the Gamification of Education. Educause Review Online, 2011.

VASSILEVA, J.; PEDRO, L. Z.; ZEM-LOPES, A. M.; ISOTANI, S. "Does Gamification Work for Boys and Girls? An Exploratory Study with a Virtual Learning Environment". In Proceedings of the 30th Annual ACM Symposium on Applied Computing. ACM. New York, NY, USA, 2015. pp. 214-219.

WERBACH, K.; HUNTER, D. For the win: How game thinking can revolutionize your business. Wharton Digital Press, 2012. 